\title{
Stabilization of Underactuated Spacecraft Dynamics via Singularly Perturbed Feedback Linearization
}

\author{
Abdulrahman H. BAJOdah \\ Aeronautical Engineering Department, \\ College of Engineering, King Abdulaziz Univ., Jeddah, Saudi Arabia \\ E-mail: abajodah@kau.edu.sa
}

\begin{abstract}
The concept of control by feedback linearization is extended to underactuated spacecrafts, by singularly perturbing the nonrealizable linear system resulting from the feedback linearizing transformation, and by utilizing the control coefficient pseudoinverse design methodology that has been recently developed. The underactuated Euler's system of dynamical equations for the spacecraft is partitioned into actuated and unactuated subsystems, and a function of the angular velocities about the unactuated body axes is used to prescribe a desired second-order dynamics for the unactuated subsystem. The evaluation of this dynamics along the trajectories defined by the underactuated Euler's dynamical equations results in a relation that is pointwise-linear in the control variables, and a condition is derived based on this relation to assess the realizability of the desired unactuated dynamics by checking the rank of the involved control coefficient Jacobian. The control variables are solved by utilizing the Moore-Penrose pseudoinverse of the control coefficient, resulting in a control law that is composed of particular and auxiliary parts. The particular part works to realize the desired linear unactuated dynamics, and the pseudo-control vector in the auxiliary part is chosen to yield a singularly perturbed feedback linearization for the actuated subsystem. The singularity avoidance problem that is related to using the control coefficient pseudoinverse is solved by modifying the pseudoinverse definition in the vicinity of the singularity, and the large control effort reduction problem is solved by deactivating the particular part of the control law whenever the angular velocities about the actuated axes become high. The control law yields a global asymptotic stability of the origin for the closed loop system when the spacecraft is equipped with two independent gas jet actuators. We show that perturbed feedback linearization is not applicable when the degree of actuation is one.
\end{abstract}

KEY WORDS: perturbed feedback linearization, pseudoinverse control, null space parametrization, underactuated dynamics. 


\section{Introduction}

The controllability of underactuated spacecrafts under different degrees and types of actuation was investigated in the seminal article by Crouch [1], and has continued to gain interest in the past two decades. The cascaded nature of Euler's model for angular motion allows one to divide the problem of spacecraft control into two parts. The first part, the focus of this article, deals with the dynamics of the spacecraft and aims at stabilizing its angular velocity components. The second part deals with the kinematics of the spacecraft and aims at driving its attitude variables to their desired values. An extensive survey of the corresponding results for underactuated spacecrafts is found in Ref. [2].

The underlying feature of underactuated dynamics is that it is not controllable by feedback linearization [3]. Some extensions have been made to feedback linearization in order to adopt underactuated systems. Examples are partial $[4,5]$ and non-regular [6] feedback linearizations. However, researchers have appealed to alternative methodologies to control underactuated spacecraft dynamics. Among the explored are those based on differential geometric concepts [7, 8], Lyapanov [9, 10], and energy [11] methods. The purpose of this article is to show that feedback linearization can be modified to adopt underactuated spacecraft dynamics by singularly perturbing the non-realizable linear dynamics obtained from the linearizing transformation. The primary tool is the control coefficient pseudoinverse and the control coefficient null-space parameterization of redundancy in the control authority, first introduced to control system design in Ref. [12].

The Moore-Penrose pseudoinverse has been utilized extensively in several engineering disciplines for the purpose of expressing nonuniqueness of solutions to problems where the requirements can be satisfied in more than one course of action, thanks to the capability of the null-space parameterization associated with it in analyzing redundancy. In particular, it has been used in the arena of robotics to model redundant manipulators, and to provide solutions to the accompanied redundancy resolution problems. 
Nevertheless, the common factor between all applications of this magnificent tool is that the dynamical systems are overactuated, i.e., the numbers of independent control elements are more than the numbers of degrees of freedom to be controlled. In this work, we illustrate that the Moore-Penrose pseudoinverse can be used to solve the opposite problem, i.e., as a means of controlling underactuated systems, where the numbers of degrees of freedom to be controlled exceed the numbers of independent control variables. This is motivated by the fact that the redundancy in control systems is ultimately in the control process itself. That is, if a dynamical system is controllable then there exists no unique strategy to control it, regardless of its degree of actuation. This also suggests to parameterize the redundancy in terms of the control variables, rather than in terms of velocities and accelerations, as illustrated in Ref. [13] for the case of fully-actuated spacecrafts.

The pseudoinverse-based control law at any time instant consists of auxiliary and particular parts, residing in the null space of the control coefficient and the range space of its pseudoinverse, respectively. The choice of the free vector in the auxiliary part of the solution is crucial. Although projected into the control coefficient null space, and hence does not affect the linearized dynamics, the choice of the free vector substantially affects the transient and asymptotic behaviors of the spacecraft state and control variables. Particular choices of the free vectors in the auxiliary parts (named the pseudo-control vectors) have been made in Ref. [13] to stabilize the internal dynamics of the spacecraft, benefiting from the pointwise-linear structure provided by the pseudoinverse-based control scheme, and resulting in spacecraft attitude stabilization and tracking control laws. The singularity avoidance problem associated with employing the pseudoinverse is solved by thresholding the Euclidean norm of the control coefficient whenever the singularity is approached.

In this work, we extend the above-mentioned methodology to the problem of underactuated spacecraft stabilization. We begin by splitting the underactuated Euler's system of equations into actuated and unactuated subsystems, and we provide a condition the satisfaction of 
which guarantees the capability of the control elements to realize a desired linear dynamics of the unactuated subsystem. The control coefficient pseudoinverse paradigm is used thereafter to obtain the control variables, and the challenging problem of choosing the pseudocontrol vector is solved, resulting in a perturbed feedback linearization of the actuated subsystem, and in a global asymptotic stability of the closed loop underactuated spacecraft dynamics. Finally, the limitation of the methodology in terms of the number of degrees of actuation is discussed.

The main contributions in this paper are threefold. First, the underactuated spacecraft dynamics is shown to be feedback linearizable up to an arbitrarily small singular perturbation of a prescribed linear dynamics. Second, the Moore-Penrose pseudoinverse that is traditionally used for overactuated control system allocation is used to control underactuated systems, and is applied to spacecrafts that are equipped with two gas jet pair actuators. Third, the null space parameterization of redundancy is utilized to realize desired linear dynamics of underactuated control systems, and to provide globally stabilizing feedback control solutions.

\section{Partitioned Form of Underactuated Euler's Equations of Motion}

The Euler's model of underactuated spacecraft dynamics is given by the system of differential equations

$$
\dot{\omega}=S(\omega) \omega+\tau
$$

where $\omega \in \mathfrak{R}^{3 \times 1}$ is the vector of angular velocities about the spacecraft's body-fixed axes, is given by

$$
S(\omega)=J^{-1} \tilde{\omega} J
$$

such that $J \in \mathfrak{R}^{3 \times 3}$ contains the spacecraft's moments of inertia, $\tilde{\omega}$ is a skew symmetric matrix of the form

$$
\tilde{\omega}=\left[\begin{array}{ccc}
0 & \omega_{3} & -\omega_{2} \\
-\omega_{3} & 0 & \omega_{1} \\
\omega_{2} & -\omega_{1} & 0
\end{array}\right]
$$


and $\tau \in \mathfrak{R}^{3 \times 1}$ is the scaled control vector. Let $d$ be the degree of actuation of the spacecraft, i.e., the number of independent gas jet pairs. The vectors $\omega$ and $\tau$ can be put in the partitioned forms

$$
\omega=\left[\begin{array}{ll}
\omega_{u}^{T} & \omega_{a}^{T}
\end{array}\right]^{T}, \quad \tau=\left[\begin{array}{ll}
\mathbf{0}_{u}^{T} & u^{T}
\end{array}\right]^{T}
$$

where $\omega_{u} \in \mathfrak{R}^{(3-d) \times 1}$ is the vector of angular velocities about the unactuated spacecraft's body axes, $\omega_{a} \in \Re^{d \times 1}$ is the vector of angular velocities about the actuated spacecraft's body axes, $u \in \mathfrak{R}^{d \times 1}$ is the scaled vector of available control torques, and $\mathbf{0} \in \mathfrak{R}^{(3-d) \times 1}$ contains zero elements. The matrix $S(\omega)$ is partitioned compatibly as

$$
S(\omega)=\left[\begin{array}{ll}
S_{11}(\omega) & S_{12}(\omega) \\
S_{21}(\omega) & S_{22}(\omega)
\end{array}\right]
$$

where $S_{11} \in \mathfrak{R}^{(3-d) \times(3-d)}, S_{12} \in \mathfrak{R}^{(3-d) \times d}, S_{21} \in \mathfrak{R}^{d \times(3-d)}$, and $S_{22} \in \mathfrak{R}^{d \times d}$. Hence, Euler's system that is given by Eqs. (1) splits into two coupled subsystems. The first one is unactuated, and is given by the equations

$$
\dot{\omega}_{u}=S_{11}(\omega) \omega_{u}+S_{12}(\omega) \omega_{a}
$$

and the second one is fully-actuated, and is given by the equations

$$
\dot{\omega}_{a}=S_{21}(\omega) \omega_{u}+S_{22}(\omega) \omega_{a}+u
$$

\section{Realizability of Linear Unactuated Spacecraft Dynamics}

To set a feedback linearizing transformation for the unactuated subsystem, we define a multivariable polynomial $\phi\left(\omega_{u}\right): \Re^{(3-d) \times 1} \rightarrow \Re$, such that $\phi\left(\mathbf{0}_{(3-d) \times 1}\right)=0$, and we use it to specify the stable linear secondorder dynamics

$$
\ddot{\phi}+c_{1} \dot{\phi}+c_{2} \phi=0, \quad c_{1}, c_{2}>0 .
$$

The first two time derivatives of $\phi\left(\omega_{u}\right)$ along the solutions of Euler's equations of motion (1), $\dot{\phi}$ and $\ddot{\phi}$, are given by

$$
\dot{\phi}=L_{f} \phi\left(\omega_{u}\right), \quad \ddot{\phi}=L_{f}^{2} \phi\left(\omega_{u}\right)+\frac{d}{d \omega_{a}}\left[L_{f} \phi\left(\omega_{u}\right)\right] u
$$


where $L_{f} \phi\left(\omega_{u}\right)$ and $L_{f}^{2} \phi\left(\omega_{u}\right)$ are the first and second Lie derivatives [14] of $\phi\left(\omega_{u}\right)$ along $f=S(\omega) \omega$. With $\dot{\phi}$ and $\ddot{\phi}$ given by Eqs. (9), it is possible to write Eq. (8) in the pointwise-linear form

$$
A(\omega) u=B(\omega),
$$

where $A(\omega) \in \mathfrak{R}^{1 \times d}$ is given by

$$
A(\omega)=\frac{d}{d \omega_{a}} L_{f} \phi\left(\omega_{u}\right)
$$

and $B(\omega) \in \mathfrak{R}^{1 \times 1}$ is given by

$$
B(\omega)=-L_{f}^{2} \phi\left(\omega_{u}\right)-c_{1} L_{f} \phi\left(\omega_{u}\right)-c_{2} \phi\left(\omega_{u}\right) .
$$

The row vector is the control coefficient relative to $\phi$ of the stable dynamics, Eq. (8), along spacecraft trajectories, and the scalar $B(\omega)$ is the corresponding control load.

Definition 1. The dynamics given by Eq. (8) is said to be realizable by the underactuated Euler's system of equations at some value of $\omega$ if there exists a control vector $u$ that solves Eq. (10) at that value of $\omega$. If this is true for all $\mathfrak{R}^{3 \times 1} \neq 0_{3 \times 1}$, then the dynamics given by Eq. (8) is said to be globally realizable by the underactuated Euler's system of equations.

Proposition 1. If the dynamics given by Eq. (8) is globally realizable by the underactuated Euler's system of equations, then

$$
A(\omega)=0_{1 \times d} \Leftrightarrow \omega=0_{3 \times 1}
$$

Proof. The existence of a vector $u$ that solves Eq. (10) at a specific value of $\omega$ is equivalent to the fact that $B$ is in the range space of $A$ at that value of $\omega$. This is possible for all values of $B$, provided that not all elements of $A$ vanish at that value of $\omega$, for which the equation is said to be consistent. Therefore, the existence of $\omega \neq 0_{3 \times 1}$ such that $A$ vanishes implies that the dynamics given by Eq. (8) is not realizable at that value of $\omega$, which violates the global realizability of the dynamics given by Eq. (8), proving sufficiency. Necessity follows from the fact that the elements of $A$ are multivariable polynomials in the components of $\omega$ with no constant terms. Hence, the evaluation of $\omega=0_{3 \times 1}$ yields $A(\omega)=0_{1 \times d}$. 
Definition 2. The zero actuated state Jacobian of the control coefficient is defined as the square matrix resulting from differentiating the control coefficient with respect to $\omega_{a}$, evaluated at $\omega_{a}=0_{d \times 1}$

$$
J_{a}\left(\omega_{u}\right)=\left[\frac{\partial A}{\partial \omega_{a}}\right]_{\omega_{a}=\mathbf{0}_{d \times 1}}
$$

The following is a necessary and sufficient condition for global realizability of the unactuated linear dynamics given by Eq. (8), based on the rank of the zero actuated state Jacobian of the control coefficient.

Proposition 2. The unactuated dynamics given by Eq. (8) is globally realizable by the underactuated Euler's system of equations if and only if

$$
\operatorname{det}\left[J_{a}\right] \neq 0 \quad \forall \omega_{u} \neq \mathbf{0}_{(3-d) \times 1}
$$

Proof. Taking the derivative of Eq. (10) with respect to $\omega_{a}$ and evaluating the resulting equation at $\omega_{a}=0_{d \times 1}$ gives

$$
J_{a} u=\left[\frac{\partial B}{\partial \omega_{a}}\right]_{\omega_{a}=\mathbf{0}_{d \times 1}}
$$

The invertibility of the zero actuated state Jacobian of the control coefficient implies that a control law $u$ can be constructed for global realizability of Eq. (8) as

$$
u=J_{a}^{-1}\left[\frac{\partial B}{\partial \omega_{a}}\right]_{\omega_{a}=\mathbf{0}_{d \times 1}}
$$

which proves necessity. Now consider the nonlinear time varying system given by the equations

$$
\dot{z}=A^{T}(z)
$$

where $z=\left[\begin{array}{ll}z_{u}^{T} & z_{a}^{T}\end{array}\right], \quad z_{u} \in \mathfrak{R}^{(3-d) \times 1}$, and $z_{a} \in \mathfrak{R}^{d \times 1}$. From proposition 1, the global realizability of the unactuated dynamics, Eq. (8), implies that the origin $z_{a}=\mathbf{0}_{d \times 1}$ is the unique equilibrium point of the system (18) at $z_{u}=\mathbf{0}_{(3-d) \times 1}$. Furthermore, since $A^{T}$ is a smooth vector field, it follows from Milnor theorem [3] that it is also a global diffeomorphism on $\mathfrak{R}^{3}$, which implies that it has continuous partial derivatives and an invertible zero actuated state Jacobian. 
Theorem 1 (Null Space Parameterization of Realized Dynamics). If the linear unactuated dynamics given by Eq.(8) along the underactuated Euler's system given by Eqs. (1) has a nonsingular zero actuated state Jacobian $J\left(\omega_{u}\right)$ of the control coefficient $A(\omega)$ for all $\omega_{u} \neq \mathbf{0}_{(3-d) \times 1}$, then the family of all controllers that realize the unactuated dynamics by the underactuated Euler's equations of motion are given by

$$
u=A^{+}(\omega) B(\omega)+P(\omega) y
$$

where " $A^{+}$" stands for the Moore-Penrose pseudoinverse of the control coefficient, and is given by

$$
A^{+}(\omega)=\frac{A^{T}(\omega)}{A(\omega) A^{T}(\omega)}, \quad A(\omega) \neq \mathbf{0}_{d \times 1}
$$

and $P(\omega) \in \Re^{d \times d}$ is the corresponding null space projection matrix

$$
P(\omega)=I_{d \times d}-A^{+}(\omega) A(\omega)
$$

and $y \in \Re^{d \times 1}$ is an arbitrarily chosen pseudo-control vector.

Proof. From proposition 1, global realizability of the unactuated linear dynamics, Eq. (8), by the underactuated system of Euler's equations implies that $A(\omega) \neq \mathbf{0}_{1 \times d} \forall \omega_{u} \neq \mathbf{0}_{(3-d) \times 1}$, at which infinite number of solutions for the pointwise linear relation (9) exist, and are linearly parameterizable by the pseudo-control vector $y$ according to Eq.(19) $[15,16]$.

The expression (19) for $u$ is composed of particular and auxiliary solutions that reside in two orthogonal subspaces. The particular solution $A^{+}(\omega) B(\omega)$ resides in the range space of $A^{+}(\omega)$, and the auxiliary solution $P(\omega) y$ resides in the null space of $A(\omega)$, where the free pseudo-control vector $y$ is projected to this space by means of the projection operator $P(\omega)$.

The choice of the pseudo-control vector $y$ does not affect realizability of the linear unactuated dynamics given by Eq. (6). Nevertheless, the choice of $y$ substantially affects stability of the actuated subsystem given by Eq. (7). In what follows, a choice of the pseudo-control vector $y$ is made to design a control coefficient 
pseudoinverse-based law, yielding a global singularly perturbed feedback linearization of the spacecraft's actuated subsystem, a global realization of the desired linear unactuated spacecraft dynamics, and a global asymptotic stability of the closed loop underactuated Euler's dynamics.

\section{Perturbed Feedback Linearizing Control}

A fundamental property of projection matrix $P(\omega)$ is that it is rank deficient. To disencumber its rank deficiency, we define the perturbed pseudoinverse of the control coefficient $A$ as [13]

$$
\tilde{A}^{+}(\omega)=\frac{1}{1+\delta} A^{+}(\omega)
$$

where $\delta$ is a nonzero scalar. The perturbed null space projection matrix $\tilde{P}(\omega)$ is defined in terms of $\tilde{A}^{+}(\omega)$ as

$$
\tilde{P}(\omega)=I_{d \times d}-\tilde{A}^{+}(\omega) A(\omega)
$$

which is of full rank [13]. The concept of perturbed null space projection matrix is crucial in the subsequent derivations of perturbed feedback linearizing control laws.

Theorem 2 (Singularly Perturbed Feedback Linearization). Let $A(\omega)$ be the control coefficient of the desired linear unactuated dynamics, Eq. (8), relative to $\phi\left(\omega_{u}\right)$ along the underactuated Euler's equations of motion, Eqs. (1), and let $B(\omega)$ be the corresponding control load. Also, let $P(\omega)$ be the projection matrix to the null space of the control coefficient $A(\omega)$, given by Eq. (21). If $\phi\left(\omega_{u}\right)$ is chosen such that

$$
\operatorname{det}\left[J_{a}(\omega)\right] \neq 0 \quad \forall \omega_{u} \neq \mathbf{0}_{(3-d) \times 1}
$$

then for any strictly stable $K \in \Re^{d \times d}$ and any perturbation $\delta>0$, the control law

$$
u=A^{+}(\omega) B(\omega)+P(\omega) y
$$

yields global asymptotic stability of the origin for the underactuated Euler's system given by Eqs. (1), where

$$
y=K \omega_{a}-S_{21}(\omega) \omega_{u}-S_{22}(\omega) \omega_{a}
$$

Proof. Consider the control law 


$$
u=A^{+}(\omega) B(\omega)+\tilde{P}(\omega) \eta
$$

obtained by replacing $P(\omega)$ by $\tilde{P}(\omega)$ in the control law $(25)$, where $\tilde{P}(\omega)$ is the perturbed projection matrix to the null space of the control coefficient $A(\omega)$, given by Eq. (23), and

$$
\eta=-\tilde{P}^{-1}(\omega)\left[S_{21}(\omega) \omega_{u}+S_{22}(\omega) \omega_{a}-K \omega_{a}+A^{+} B(\omega)\right] .
$$

Applying the feedback linearizing control law given by Eq. (27) in the actuated subsystem given by Eq. (7) yields the asymptotically stable closed loop actuated subsystem

$$
\dot{\omega}_{a}=K \omega_{a}
$$

Nevertheless, the continuity of the eigenvalues of $K$ implies that if the magnitude of $\delta$ is small enough then the control law given by

$$
u=A^{+}(\omega) B(\omega)+P(\omega) \eta
$$

yields asymptotically stable closed loop actuated subsystem also. Satisfying the condition (24) by $\phi\left(\omega_{u}\right)$ implies that applying the control law given by Eq. (30) yields exact tracking of the unactuated dynamics given by Eq. (8). Since the particular part of the control law $A^{+}(\omega) B(\omega)$ resides in the range space of $A^{+}(\omega)$, the last term of the pseudo-control vector $\eta$ given by $h=-\tilde{P}^{-1}(\omega) A^{+}(\omega) B(\omega)$ also resides in the range space of $A^{+}(\omega)$. Therefore, $h$ is orthogonal to the null space of $A(\omega)$, i.e.,

$$
P(\omega) h=-P(\omega) \tilde{P}^{-1}(\omega) A^{+}(\omega) B(\omega)=\mathbf{0}
$$

and the expression (30) for $u$ becomes

$$
u=A^{+}(\omega) B(\omega)-P(\omega) \tilde{P}^{-1}(\omega)\left[S_{21}(\omega) \omega_{u}+S_{22}(\omega) \omega_{a}-K \omega_{a}\right] .
$$

With the feedback control law given by Eq. (32), the closed loop actuated subsystem becomes

$$
\dot{\omega}_{a}=S_{21}(\omega) \omega_{u}+S_{22}(\omega) \omega_{a}+A^{+}(\omega) B(\omega)-P(\omega) \tilde{P}^{-1}(\omega)\left[S_{21}(\omega) \omega_{u}+S_{22}(\omega) \omega_{a}-K \omega_{a}\right] .
$$

Nevertheless, the expression $P(\omega) \tilde{P}^{-1}(\omega)$ can be expanded as

$$
\begin{gathered}
P(\omega) \tilde{P}^{-1}(\omega)=\left[I_{d \times d}-A^{+} A\right]\left[I_{d \times d}-\frac{1}{1+\delta} A^{+} A\right]^{-1}=\left[I_{d \times d}-A^{+} A\right] \sum_{i=0}^{\infty}\left(\frac{1}{1+\delta} A^{+} A\right)^{i} \\
=\left[I_{d \times d}-A^{+} A\right]\left[I_{d \times d}+\sum_{i=1}^{\infty}\left(\frac{1}{1+\delta}\right)^{i}\left(A^{+} A\right)^{i}\right]=\left[I_{d \times d}-A^{+} A\right]\left[I_{d \times d}+A^{+} A \sum_{i=1}^{\infty}\left(\frac{1}{1+\delta}\right)^{i}\right]
\end{gathered}
$$




$$
\begin{gathered}
=\left[I_{d \times d}-A^{+} A\right]+\left[A^{+} A-A^{+} A A^{+} A\right] \sum_{i=1}^{\infty}\left(\frac{1}{1+\delta}\right)^{i} \\
=\left[I_{d \times d}-A^{+} A\right]+\left[A^{+} A-A^{+} A\right] \sum_{i=1}^{\infty}\left(\frac{1}{1+\delta}\right)^{i} \\
=\left[I_{d \times d}-A^{+} A\right]=P(\omega)
\end{gathered}
$$

Therefore, the expression (33) for $\dot{\omega}_{a}$ can be written as

$\dot{\omega}_{a}=S_{21}(\omega) \omega_{u}+S_{22}(\omega) \omega_{a}+A^{+}(\omega) B(\omega)+P(\omega) \tilde{P}^{-1}(\omega)\left[K \omega_{a}-S_{21}(\omega) \omega_{u}-S_{22}(\omega) \omega_{a}\right]$,

which is obtained by using the expression (26) for $y$ in the control law given by Eq. (25), and applying it to the actuated subsystem given by Eq. (7).

Remark The resulting closed loop system is singularly perturbed from the non-realizable linear system

$$
\ddot{\phi}+c_{1} \dot{\phi}+c_{2} \phi=0, \quad \dot{\omega}_{a}=K \omega_{a}
$$

obtained by replacing $P(\omega)$ by $\tilde{P}(\omega)$ in the control law (25).

\section{Singularity Avoidance and Control Effort Reduction}

According to proposition 1, the control coefficient $A$ must become singular when the components of the spacecraft angular velocity vector vanish, which implies from Eq. (20) that the control coefficient pseudoinverse $A^{+}$must go unbounded as the spacecraft detumbles. This problematic phenomenon is well known in the pseudoinverse applications when the elements of $A$ are functions of the motion variables, and several remedies are given in the pseudoinverse literature. In the current context, the control coefficient singularity is a source of instability for the closed loop system because it causes the vector field given by Eq. (35) to become unbounded. To limit the growth of the control coefficient pseudoinverse, it is proposed in Ref. 13 for the case of 
fully-actuated spacecraft control to modify the definition of the pseudoinverse given by Eq. (20) to be

$$
A_{m}^{+}(\omega)= \begin{cases}\frac{A^{T}(\omega)}{A(\omega) A^{T}(\omega)} & : A(\omega) A^{T}(\omega)>\beta \\ \frac{A^{T}(\omega)}{\beta} & : A(\omega) A^{T}(\omega) \leq \beta\end{cases}
$$

where $\beta$ is a small positive scalar. The same method for singularity avoidance is adopted in this paper for the case of underactuated spacecraft control. Another problem that is related to pseudoinverse control is the large control effort needed when the magnitude of the control load $B(\omega)$ is large. This causes the particular part of the control law and the angular velocities about the actuated axes to go unacceptably high before detumbling. For this reason, we propose to modify the control law (25) such that to deactivate the particular part whenever the Euclidean norm of $\omega_{a}$ becomes large compared to the Euclidean norm of $\omega_{u}$ according to the relation

$$
u=A_{m}^{+} B \operatorname{step}\left(\left\|\omega_{\mathrm{u}}\right\|-\varepsilon\left\|\omega_{a}\right\|\right)+P(\omega) y
$$

where $\varepsilon$ is a small positive number, and the step function is defined as

$$
\operatorname{step}(x)=\left\{\begin{array}{l}
1: x>0 \\
0: x<0
\end{array}\right.
$$

This modification implies that the portion of the control law that drives the desired unactuated dynamics is disabled whenever the magnitudes of the angular velocities about the actuated axes become high relative to those about the unactuated axes.

Remark Thresholding the control coefficient pseudoinverse according to Eq. (37) implies that the desired unactuated dynamics that is given by Eq. (8) is realized only when $A(\omega) A^{T}(\omega) \geq \beta$ where the true definition of the pseudoinverse applies. However, the angular velocity components about the unactuated axes can be brought arbitrarily close to the origin according to Eq. (8) by reducing the value of $\beta$.

Remark Deactivating the particular part of the control law according to the relative magnitudes of $\omega_{\mathrm{u}}$ and $\omega_{a}$ implies that the control law given by 
Eq. (38) can become discontinuous, depending on the initial condition of $\omega$, which results in nonsmooth trajectories of the spacecraft angular velocity components.

\section{Perturbed Feedback Linearizability Based on the Degree of Actuation}

\section{Case 1: Spacecraft with Two Degrees of Actuation ( $d=2)$}

The spacecraft is stabilizable with the two actuators mounted along two principal axes, with no symmetry about the unactuated body axis [7]. The unactuated body axis is the one about which the spacecraft angular velocity component is $\omega_{1}$. Since the control coefficient is a single raw matrix, the range space of its pseudoinverse has the dimension one. Hence, the null space of the control coefficient $A$ has the dimension $d-1=1$, and the pseudo-control vector $y$ can be chosen according to Eq. (26) for perturbed feedback linearization of the actuated dynamics.

\section{Case 2: Spacecraft with One Degree of Actuation $(d=1)$}

The spacecraft is stabilizable with one actuator not mounted on a principal axis,9 with the possibility of inertial symmetry [10]. The unactuated body axes are the ones about which the spacecraft angular velocity components are $\omega_{1}$ and $\omega_{2}$. The range space of the control coefficient is a scalar in this case, and the null space projection matrix given by Eq. (21) becomes

$$
P(\omega)=1-\frac{A(\omega)}{A(\omega)}=0
$$

which implies that the null space of the control Jacobin $A$ has the dimension zero, and that the auxiliary part of the control law (25) vanishes. Hence, the pseudo-control vector $y$ cannot be constructed for perturbed feedback linearization of the actuated dynamics.

\section{Control System Design Procedure}

The procedure of control system design for singularly perturbed feedback linearization of underactuated spacecraft dynamics is summarized in the following steps: 
1. The function $\phi\left(\omega_{1}\right)$ is chosen such that

(a) $\phi(0)=0$.

(b) The control coefficient $A(\omega)$ given by Eq.(11) has actuated state Jacobian

$$
J_{a}\left(\omega_{1}\right)=\left[\frac{\partial A}{\partial \omega_{a}}\right]_{\omega_{a}=\mathbf{0}_{2 \times 1}}
$$

that is nonsingular for all $\omega_{1} \neq 0$.

2. The coefficients $c_{1}$ and $c_{2}$ in the desired linear dynamics given by Eq. (8) are chosen such that $\phi$ is stable. This implies that both $c_{1}$ and $c_{2}$ are strictly positive.

3. The control law is given by Eq. (38), where $A_{m}^{+}$is given by Eq. (37), $B(\omega)$ is given by Eq. (12), and $P(\omega)$ is given by Eq.(21). The pseudocontrol vector $y$ is given by Eq. (26), where $K \in \mathfrak{R}^{2 \times 2}$ is a strictly stable matrix, $\omega_{u}=\omega_{1}, \omega_{a}=\left[\begin{array}{ll}\omega_{2} & \omega_{3}\end{array}\right]^{T}, S_{21} \in \mathfrak{R}^{2 \times 1}$, and $S_{22} \in \mathfrak{R}^{2 \times 2}$ are partitions of the matrix $S(\omega)$ given by Eq. (5).

\section{Numerical Simulations}

The control coefficient $A(\omega)$ relative to $\phi\left(\omega_{u}\right)=\omega_{1}^{2}$ is

$$
A(\omega)=\left[\frac{2\left(I_{22}-I_{33}\right)}{I_{11}} \omega_{1} \omega_{3} \frac{2\left(I_{22}-I_{33}\right)}{I_{11}} \omega_{1} \omega_{2}\right]
$$

and its zero actuated state Jacobian is

$$
J_{a}\left(\omega_{1}\right)=\left[\frac{\partial A^{T}(\omega)}{\partial \omega_{2}} \frac{\partial A^{T}(\omega)}{\partial \omega_{3}}\right]_{\substack{\omega_{2}=0 \\
\omega_{3}=0}}=\left[\begin{array}{ll}
0 & 2\left(I_{22}-I_{33}\right) \\
I_{11} & \frac{2\left(I_{22}-I_{33}\right)}{I_{11}} \omega_{1}
\end{array}\right]
$$

which is nonsingular for all $\omega_{1} \neq 0$, implying that Eq. (8) is globally realizable by the underactuated Euler's equations. For a spacecraft with three principal moments of inertia ( in $\mathrm{Kg}-\mathrm{m}^{2}$ ) $I_{11}=10, I_{22}=6.3, I_{33}=8.5$, Figs. (1) and (2) show the resulting angular velocities about the three principal axes and the required control variables, where the matrix $K$ is 
taken to be diagonal with elements 0.1 and $0.2, c_{1}=2, c_{2}=1, \beta=10^{-9}$, and $\varepsilon=10^{-7}$.

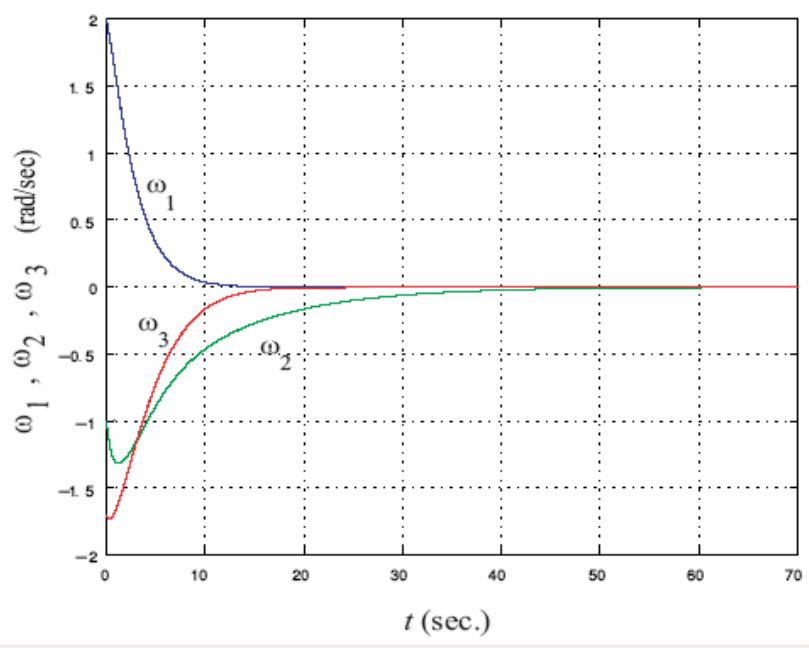

FIG. 1. Angular velocities about the three principal axes

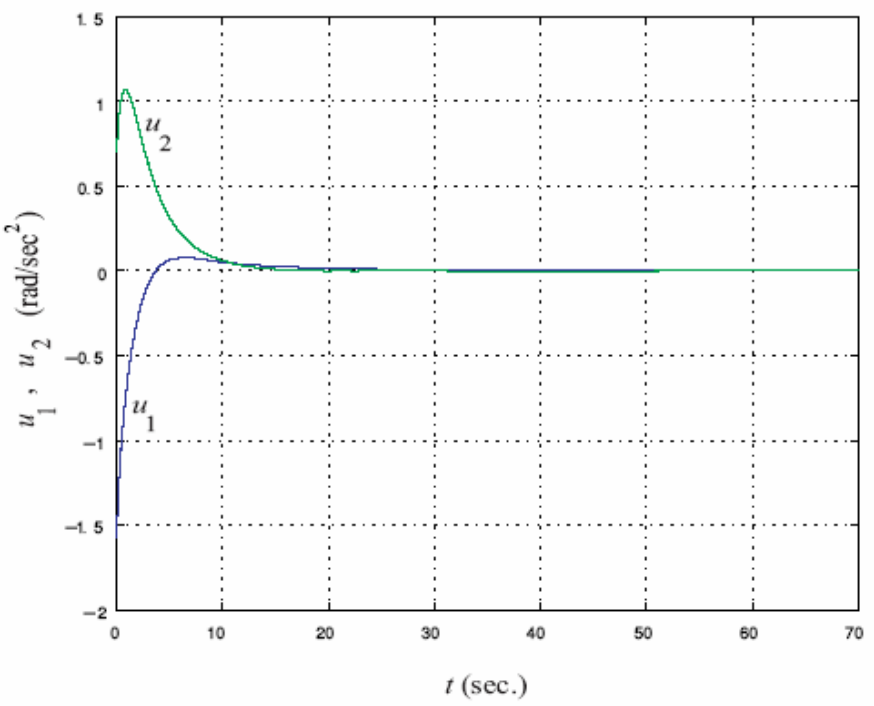

Fig. 2. Scaled control torques about the two actuated axes 


\section{Conclusions and Future Work}

The control coefficient pseudoinverse design methodology is applied to the problem of underactuated spacecraft stabilization, resulting in feedback control systems that are linear in the angular velocity about the unactuated axis and singularly perturbly linear in the angular velocities about the actuated axes, and in closed loop trajectories that are globally asymptotically stable. Nevertheless, numerical issues that are related to the particular part of the control law arise, and the control law is modified accordingly, including thresholding the pseudoinverse whenever the singularity is approached, and deactivating the particular part of the control law when the angular velocities of the spacecraft about the actuated axes become large. Optimizing the control law performance for these issues and extending the methodology to underactuated spacecraft attitude control are ongoing research efforts by the author.

\section{References}

[1] Crouch, P. E., "Spacecraft attitude control: Applications of geometric control theory to rigid body models," IEEE Transactions on Automatic Control, Vol. 29, No. 4, pp. 321-331, (1984).

[2] Tsiotras, P. and Doumtchenko, V., "Control of spacecraft subject to actuator failure: Stateof-the-art and open problems," Journal of the Astronautical Sciences, Vol. 48, No. 2-3, pp. 337-358, (2000).

[3] Isidori, A., Nonlinear Control Systems, Springer, 3rd ed., (1995).

[4] Spong, M., "Partial feedback linearization of underactuated mechanical systems," IEEE international conference on intelligent robots and systems, Vol. 1, pp. 314-321, (1994).

[5] Kaustubh, P., Jaume, F., and Agrawal, S., "Velocity control of a wheeled inverted pendulum by partial feedback linearization," IEEE Transactions on Robotics, Vol. 21, No. 3, pp. 505-513, (2005).

[6] Ge, S., Sun, Z., Lee, T., and Spong, M., "Feedback linearization and stabilization of secondorder non-holonomic chained systems," International Journal of Control, Vol. 74, No. 14, pp. 1383-1392, (2001).

[7] Brockett, R. W., "Asymptotic Stability and Feedback Stabilization," Differential Geometric Control Theory, edited by R.W. Brockett, R.S. Millman, and H.J. Sussman, Boston, pp. 181-208, (1983).

[8] Aeyels, D., "Stabilization by smooth feedback of the angular velocity of a rigid body," Systems \& Control Letters, Vol. 5, pp. 59-63, (1985).

[9] Aeyles, D. and Szafranski, M., "Comments on the stabilizability of the angular velocity of a rigid body," Systems \& contol Letters, Vol. 10, pp. 35-39, (1988). 
[10] Sontang, E. and Sussmann, H., "Further comments on the stabilizability of the angular velocity of a rigid body," Systems \& Control Letters, Vol. 12, pp. 213-217, (1988).

[11] Outbib, R. and Sallet, G., "Stabilizability of the angular velocity of a rigid body revisited," Systems \& Control Letters, Vol. 18, pp. 93-98, (1992).

[12] Bajodah, A. H., Hodges, D. H., and Chen, Y.-H., "Inverse dynamics of servo-constraints based on the generalized inverse," Nonlinear Dynamics, Vol. 39, No. 1-2, pp. 179-196, (2005).

[13] Bajodah, A. H. and Hodges, D. H., "Linear parametrization of nonlinear spacecraft control by pseudoinversion of the control coefficient," Proceedings of the AIAA conference of Guidance, Navigation, and Control, San Francisco, (2005).

[14] Slotine, J. E. and Li, W., Applied Nonlinear Control, Prentice-Hall, Englewood Cliffs, NJ, (1991).

[15] Udwadia, F. E. and Kalaba, R. E., Analytical Dynamics, A New Approach, Cambridge University Press, New York, NY, (1996).

[16] Bernstein, D., Matrix mathematics: theory, facts, and formulas with application to linear system theory, Princeton University Press, (2005). 


\section{الموازنة الآلية لايناميكا مركبة الفضاء تحت المشغَّــة بواسطة

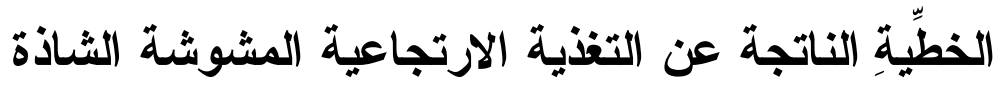

\section{عبد الرحمن حسن باجودة}

قسم هندسة الطبران، كلية الهندة، جامعة الملك عبد العزبز

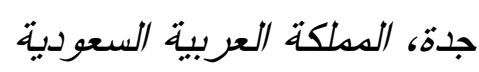

ينبني البحث على الطريقة الني نم استحداثها مؤخر اء للموازنة الآلية

لمركبات الفضاء. من المعلوم أن جسماً صلبا كمركبة الفضاء يمكن

موازنة حركته الزاوية ( أي إيقافها ) بواسطة ثلاثة عزوم حول

محاوره الرئيسية الثالثة. لكن المشوّق أن نعلم أنَّ الجسم قابل أيضاً

للموازنة الآلية بالاستغناء عن أحد هذه العزوم و الإبقاء على اثثين

منها فقط، بشرط ألا يكون عزما القصور الذاتي حول المحورين

المشغََّيَنْ منساويسين. تتبنسَّى طريقة المعكوس الثبيه لمر افق التحكم

هذه العملية عـن طريق تعريف دالة موجبة معرَّفة في مربَّع

مركَّبّة السرعة الزاوية للمركبة حول المحور الرئيسي غير

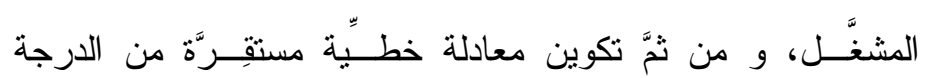

الثانية في الدالة المعرَّفة و حلــــهُها لإيجـــاد قيم عزوم التــَّكم 
بواسطة قلب مرافق التحكم بطريقة شبه المعكو. يتكون قانون التحكم بالتغذية الرجعية الناتج من جزئين اثثين، أحدهما مخصص و الأخر تكميلي. الجزء المخصص يعمل على اقتفاء السرعة الزاوية للمركبة الفضائية حول المحور الرئيسي غير المشغَّـل حسب المعادلة الخطـيّة، و والجزء التكميلي يحتوي على متغير خطيٍٍ يجري استغلاله لتفعيل الخطـيّة الثشاذة في ديناميكا السرعة الزاوية حول المحورين المشغلين بحيث تكون ديناميكا المركبة مستقرةً. المشكلة المألوفة في تطبيقات المعكوس المعدم هي عدم الاسنقرار الرقمي الناتج عن اقتراب قيمة مقام المعكوس المعدم من الصفر مع اقتراب قيم متغيرات المسألة من القيم المطلوبة، ونعالجها بتعديل تعريف المعكوس المعمم كلما تلاشى مقامه بــهذف تلافي الصفر. نتائج التشبيهات الرقمية تظهر كفاءة الطريقة المقدَّمة في التحكم الآلي بمركبات الفضاء، وكطريقةٍ جديدةٍ للتحكم بالتغذية الاسترجاعية، تجمع بين سهولة التحكم الخطي وفاعلية التحكم اللاخطي. 